\title{
Tecnologia assistiva e tradução para Libras: desafios da ferramenta de tradução automática de vídeos VLibras
}

\author{
Assistive technology and translation into Libras: challenges of the VLibras video machine \\ translation tool \\ Tecnología de asistencia y traducción en Libras: retos de la herramienta de traducción automática \\ de vídeos VLibras
}

Recebido: 16/09/2021 | Revisado: 21/09/2021 | Aceito: 23/09/2021 | Publicado: 24/09/2021

\author{
Claudemir Jeremias de Lima \\ ORCID: https://orcid.org/0000-0001-9497-4348 \\ Universidade Federal Rural de Pernambuco, Brasil \\ E-mail: Claudemir.jlima@ufrpe.br \\ Elidiene Gomes de Oliveira Lima \\ ORCID: https://orcid.org/0000-0002-3625-4817 \\ Universidade Federal de Pernambuco, Brasil \\ E-mail: elidienegomes@hotmail.com \\ Neiton Carvalho da Silva \\ ORCID: https://orcid.org/0000-0001-7786-856X \\ Universidade Federal Rural de Pernambuco, Brasil \\ E-mail: nsistema@gmail.com \\ Jorge da Silva Correia Neto \\ ORCID: https://orcid.org/0000-0001-9977-1267 \\ Universidade Federal Rural de Pernambuco, Brasil \\ E-mail: jorgecorreianeto@gmail.com
}

\begin{abstract}
Resumo
A educação é um dos habilitadores para que os cidadãos entrem no mercado de trabalho. Porém, para democratizar o acesso de todos a uma educação de qualidade e inclusiva, os métodos devem ser ajustados para que as pessoas surdas, que são o alvo do presente estudo. A pesquisa buscou investigar o uso da tecnologia assistiva VLibras, que envolve um conjunto de vídeos em Língua Brasileira de Sinais (LIBRAS) para pessoas surdas. Com uso do VLibras pode-se agregar o tradutor LIBRAS às vídeo-aulas com pouco esforço de edição do vídeo. Com base em métodos qualitativos descritivos, foi realizado um levantamento bibliográfico. Os resultados mostram haver evidências de que as tecnologias assistivas contribuem para a autonomia dos alunos com necessidades especiais. E que o software VLibras é uma opção viável na construção de vídeo aulas, entretanto a necessidade do professor realizar edição no vídeo com software de edição.
\end{abstract}

Palavras-chave: Educação inclusiva; VLibras; Tecnologias assistivas.

\begin{abstract}
Education is one of the enablers for citizens to enter the job market. However, in order to democratize the access of all to a quality and inclusive education, the methods must be adjusted so that deaf people, who are the target of the present study. The research sought to investigate the use of the assistive technology VLibras, which involves a set of videos in Brazilian Sign Language (LIBRAS) for deaf people. With the use of VLibras, it is possible to add the LIBRAS translator to the video classes with little video editing effort. Based on descriptive qualitative methods, a bibliographic survey was carried out. The results show there is evidence that assistive technologies contribute to the autonomy of students with special needs. And that the VLibras software is a viable option in the construction of video classes, however the need for the teacher to perform video editing with editing software.
\end{abstract}

Keywords: Inclusive education; VLibras; Assistive technologies.

\section{Resumen}

La educación es uno de los elementos que permiten a los ciudadanos acceder al mercado laboral. Sin embargo, para democratizar el acceso de todos a una educación de calidad e inclusiva, los métodos deben ajustarse para que las personas sordas, que son el objetivo de este estudio. La investigación pretendía investigar el uso de la tecnología de asistencia VLibras, que consiste en un conjunto de vídeos en lengua de signos brasileña (LIBRAS) para personas sordas. Con el uso de VLibras es posible añadir el traductor de LIBRAS a las lecciones de vídeo con poco esfuerzo de edición de vídeo. A partir de métodos cualitativos descriptivos, se realizó un estudio bibliográfico. Los resultados muestran que hay pruebas de que las tecnologías de apoyo contribuyen a la autonomía de los alumnos con 
necesidades especiales. Y que el software VLibras es una opción viable en la construcción de lecciones de vídeo, sin embargo la necesidad de que el profesor realice la edición de vídeo con software de edición.

Palabras clave: Educación inclusiva; VLibras; Tecnologías de apoyo.

\section{Introdução}

A educação é um direito de todos, segundo o art. 205 da Constituição Federal de 1988 (CF/88) (BRASIL, 2021). No entanto, a educação inclusiva continua sendo o principal desafio dos profissionais da educação, desde a gestão escolar, que garante ou não a matrícula dos alunos, até a metodologia utilizada pelos professores no ensino presencial. Visando apoiar esse desafio, algumas políticas públicas têm se baseado nas TIC como suporte para pessoas com deficiência ou necessidades específicas, sendo as tecnologias assistivas um exemplo, já que apoiam o processo de ensino-aprendizagem de pessoas com necessidades específicas (De Lima, 2021).

A tecnologia utilizada na reabilitação ou no aumento da capacidade funcional das pessoas com deficiência é chamada Tecnologia Assistiva (TA) (Federici \& Scherer, 2018). A TA é um campo do conhecimento que objetiva promover ou capacitar pessoas com deficiência funcional. São tecnologias usadas para identificar recursos e serviços que ajudam a fornecer ou expandir as capacidades funcionais de pessoas com deficiência, promovendo assim uma vida independente e inclusiva (bersch \& tonolli, 2006).

Segundo Edyburn (2015), é possível que essas pessoas vivam, estudem e trabalhem por conta própria através de tecnologias que reduzem, eliminam ou minimizam o impacto das dificuldades, ou deficiências existentes. Segundo Federici e Scherer (2018), as TA desempenham um papel fundamental na integração social de pessoas com deficiências físicas, sensoriais, de comunicação ou cognitivas. O objetivo da tecnologia assistiva é proporcionar às pessoas com deficiência maior independência, qualidade de vida e inclusão social, ampliando a comunicação, a mobilidade, o controle ambiental, o aprendizado, o trabalho e a integração com a família, amigos e sociedade, complementam os citados autores.

Uma destas TA é a VLibras, sendo uma ferramenta para tradução de conteúdos em português para a língua brasileira de sinais, a LIBRAS. A VLibras faz parte das políticas de inclusão do governo federal e está disponível para download em https://www.gov.br/governodigital/pt-br/vlibras/.

Nessa perspectiva, o presente estudo buscou responder a seguinte pergunta de pesquisa: quais são os principais desafios da tecnologia assistiva VLibras na tradução para Libras dos vídeos gerados por professores? Assim, o artigo objetiva investigar os principais desafios da tecnologia assistiva VLibras na tradução para Libras dos vídeos gerados por professores.

Com isso, a partir dessa seção introdutória, o presente artigo está estruturado em seis seções. A segunda seção traz uma revisão de literatura sobre TA, língua de sinais, software VLibras e ainda edição de vídeos. Na seção seguinte são apresentados alguns trabalhos relacionados com o contexto investigado e que apoiaram a realização desta pesquisa. Em seguida é apresentado o percurso metodológico trilhado pelo estudo. Na quinta seção são discutidos os Resultados da pesquisa e por fim são apresentadas as Considerações Finais.

\section{Trabalhos Relacionados}

Para posicionar nosso artigo e indicar as suas contribuições, esta seção apresenta os trabalhos relacionados.

Araújo et al. (2021) realizaram uma revisão sistemática com o objetivo de verificar o impacto das várias formas de TA existentes na reabilitação de pessoas com dislexia. Nos artigos estudados havia um total de 344 participantes com dislexia, dos quais 323 participantes eram crianças e adolescentes e 21 eram jovens e adultos. Os estudos mostram que as TA têm um efeito positivo na leitura e reforçam a importância das TA para pessoas com dislexia.

Já De Lima et al. (2021) afirmam que, para tornar a educação acessível a todos de forma qualitativa e inclusiva, os 
métodos devem ser ajustados, e que a educação a distância (EAD) é uma opção que pode potencializar a autonomia do aluno.

Castro (2019) discute o uso do VLibras no ensino das quatro operações básicas de matemática no ensino secundário. O objetivo foi formar alunos surdos e ouvintes de uma escola de ensino fundamental que, com o auxílio da ferramenta educacional VLibras, utilizaram LIBRAS no aprendizado das quatro operações básicas. A pesquisa mostrou que essa TA contribuiu positivamente para a educação.

Da Mata Caetano et al. (2017) analisam as contribuições que os aplicativos VLibras e Hand Talk podem trazer para o processo de ensino-aprendizagem de crianças com deficiência auditiva em uma escola no Espírito Santo. Está dividido em três etapas: investigações preliminares com as escolas, sugestões de uso dos aplicativos VLibras e Hand Talk nas escolas e avaliação das sugestões. $\mathrm{O}$ estudo constatou que os aplicativos utilizados produziram resultados positivos no processo de inclusão e construção do conhecimento dos alunos.

Souza et al. (2021), analisaram duas ferramentas de tecnologias assistivas: "WIKILIBRAS" e "VLIBRAS". A questão que norteia a pesquisa tenta investigar: Como as agências governamentais podem promover o acesso de alunos surdos-mudos a ferramentas de tecnologia assistiva por meio de políticas e programas educacionais? Portanto, o objetivo deste trabalho é analisar os recursos de tecnologia assistiva fornecidos pelo governo nos níveis municipal, estadual e federal, e analisar o auxílio que eles fornecem para a aprendizagem de crianças surdas em escolas regulares. Uma tabela de análise foi construída para poder identificar certos elementos pertencentes a cada elemento, por exemplo, identificar seu executor, sua função, público-alvo e acessórios necessários para a utilização do programa. Ambos são projetados para serem úteis aos surdos e beneficiar pessoas com audição, pois proporcionam acesso totalmente gratuito para quem deseja estudar ou se comunicar em Libras. A julgar pela conclusão desta pesquisa, os arquivos selecionados "VLIBRAS" e "WIKILIBRAS" são muito úteis para surdos na pronúncia, pois promovem práticas inclusivas no ambiente social e escolar

Por fim, Braga (2020) descreve a relação entre surdos e ouvintes e tradutores automáticos de língua de sinais, e investiga a utilização dessas ferramentas como serviços de tradução de sites da Internet. O uso cada vez maior de dispositivos eletrônicos de acesso à Internet e o desenvolvimento de ferramentas de tradução automática, fáceis de usar e quase sempre gratuitas, tornam possível o uso dessas ferramentas, e sua expansão é esperada, até porque podem ser utilizadas pelos usuários. No entanto, questões relacionadas a limitações no processo de tradução têm desencadeado algumas discussões sobre sua eficácia. De acordo com os dados coletados pela comparação cega da tradução homem-máquina, após o processamento estatístico, os resultados mostram que, além de explicar a relação entre o ouvinte fluente de Libras e o surdo, diferentes fatores afetam a frequência de uso e o impacto dessa técnica.

\section{Fundamentação Teórica}

Essa seção apresenta os conceitos para uma melhor compreensão deste trabalho. Inicialmente, são apresentados os conceitos Tecnologias assistivas. Em seguida, são mostradas as características da Língua de sinais e são apresentados alguns conceitos básicos da tecnologia assistiva VLibras. Por fim, conceitos sobre software de edição.

\subsection{Tecnologias Assistivas}

As tecnologias assistivas conformam uma área do conhecimento multifacetada. Vão desde uma ferramenta básica de suporte (como a comunicação) até a configuração de um novo ambiente de aquisição de conhecimento e ampliação do relacionamento interpessoal. O Comitê de Ajudas Técnicas (CAT) propôs o conceito de tecnologia assistiva como área do conhecimento e apontou suas características.

Tecnologia assistiva é uma área do conhecimento, de característica interdisciplinar, que engloba produtos, recursos, 
metodologias, estratégias, práticas e serviços que objetivam promover a funcionalidade, relacionada à atividade e participação de pessoas com deficiência, incapacidades ou mobilidade reduzida, visando sua autonomia, independência, qualidade de vida e inclusão social (CAT, 2006, p. 9).

Portanto, a TA surgiu para minimizar as barreiras de pessoas com deficiência ou dificuldades de mobilidade. Por isso, as políticas públicas devem proporcionais recursos materiais, recursos humanos, equipamentos e meios legais para que os deficientes possam se tornar cidadãos como qualquer outra pessoa, e ter possibilidades específicas de usufruir o que a sociedade prevê para sua integração nas escolas.

As TA, como destacam Sartoretto e Bersch (2021), englobam recursos e serviços. Entendem-se recursos como os equipamentos (ou parte deles), produtos ou sistemas fabricados em série ou sob medida, utilizado para aumentar, manter ou melhorar as capacidades funcionais das pessoas com deficiência; já os serviços são aqueles que diretamente auxiliam uma pessoa com deficiência a selecionar, comprar ou usar os recursos acima definidos, complementam os citados autores.

Diversos recursos tecnológicos digitais têm sido desenvolvido, uma dessas TA é a que realiza tradução de textos em áudio, mas vale ressaltar também a que traduz textos para a Língua Brasileira de Sinais, LIBRAS, usando avatares. São exemplos dessas TA o VLibras, o Hand Talk e o ProDeafe Rybená, que podem ser usados em várias plataformas, como software de computador, plug-ins de navegador de Internet para tradução de sites e aplicativos móveis.

De fato, com o apoio das TIC, existe uma série de alternativas no campo das TA que podem auxiliar os alunos surdos no processo de aprendizagem, conforme a situação específica. Por isso é importante a formação de professores para o uso dessas TA. Além disso, ao se discutir a educação inclusiva de surdos (foco desta pesquisa), é preciso considerar suas necessidades, como acolhimento, mas também em termos de desenvolvimento global (Da Silva, 2021).

\subsection{A Língua de Sinais}

Segundo Bogas (2014), nossa história da língua de sinais se confunde com a história dos surdos e mudos brasileiros. Até o século XV, os surdos em todo o mundo eram considerados ineducáveis. A partir do século XVI, isso começa a mudar na Europa, com a luta pela educação de surdos. A convite de Dom Pedro II, um surdo francês chamado Édouard Huet du Pavillon veio ao Brasil e fundou a primeira escola de surdos do país, o Colégio Imperial para Surdos, atualmente Instituto Nacional de Educação de Surdos (INES).

Com a persistência no uso e a crescente exploração da legitimidade da língua de sinais, a LIBRAS foi mais uma vez aceita e a luta pelo seu reconhecimento não parou. Em 1993, uma nova guerra começou, pois o projeto de lei que visava regulamentar o idioma do país só conseguiu ser aprovado em 2002. Em termos de oferta pela rede educacional, a lei $\mathrm{n}^{\circ}$ 10.436/2002 afirma, em seu art. 4, que

O sistema educacional federal e os sistemas educacionais estaduais, municipais e do Distrito Federal devem garantir a inclusão nos cursos de formação de Educação Especial, de Fonoaudiologia e de Magistério, em seus níveis médio e superior, do ensino da Língua Brasileira de Sinais - LIBRAS, como parte integrante dos Parâmetros Curriculares Nacionais - PCN, conforme legislação vigente (BRASIL, 2002, online).

Com esse amparo legal a LIBRAS passa a fazer parte do currículo escolar, mas ainda existem obstáculos para a implementação da lei. Mesmo sem apoio financeiro, as pessoas estão dispostas a lutar pelo cumprimento da lei para dar aos alunos surdos o direito de receber uma educação digna, ou pelo menos o direito similar dos ouvintes. LIBRAS é uma língua natural, mas também há suas complexidades para se aprender. Para a estrutura dos sinais, é muito importante analisar a estrutura fonética da LIBRAS, que tem as seguintes características: configuração das mãos, articulações, movimentos, orientação e expressões faciais (Castro, 2019).

Ramos (2021, p. 15) destaca, sobre a Lei e Decreto sobre a Língua Brasileira de Sinais, que: 
Com a difusão da Língua Brasileira de Sinais - Libras no território brasileiro, impulsionada pelos aparatos legais como a Lei 10.436/2002 e o Decreto 5.626/2005, a preocupação com a formação, ensino e a garantia de conhecimentos específicos para as pessoas surdas e ouvintes vêm se tornando objetos de pesquisas significativas para compreender a necessidades da comunidade surda. A Língua Brasileira de Sinais é uma língua reconhecida, de acordo com a Lei ${ }^{\circ}$ 10.436/2002, como a língua oficial da comunidade surda brasileira. É caracterizada por utilizar de um espaço-visual, organizando seus referentes para a transmissão da mensagem na comunicação.

Braga (2020) faz algumas observações sobre a tradução da tradução automática e língua de sinais pelos softwares de tecnologia assistiva. No Brasil, os usuários de LIBRAS (Língua Brasileira de Sinais) constituem uma minoria linguística que busca obter informações para a utilização de seus direitos civis. Uma vez que a maioria dos ouvintes que usam o português como língua oficial, alguns esforços têm sido feitos e algumas iniciativas têm sido propostas para fornecer soluções em forma de software e propor a eliminação das barreiras linguísticas existentes.

Por possuir uma forma visual espacial, a língua de sinais apresenta características distintas da língua de escuta oral, o que representa um desafio para a construção do mecanismo de análise de dados da língua. O movimento das mãos, braços, cabeça e tronco no espaço ao redor do remetente - sua percepção é realizada por meio da visão. Isso torna a automação da tradução difícil. Além disso, existem diferenças sutis na execução de símbolos relacionados à construção de significados, como o uso de expressões não artificiais relacionadas a essas linguagens. Mesmo diante do desafio descrito anteriormente, vários esforços têm sido aplicados para traduzir automaticamente para a língua de sinais LIBRAS.

\subsection{A Tecnologia Assistiva VLibras}

Esta TA permite a criação de softwares que auxiliam no desenvolvimento de alunos surdos-mudos, incorporando a língua de sinais para que os alunos possam entender o que está acontecendo na tela do computador ou outros dispositivos móveis, sem ter a necessidade de um tradutor ao seu lado para traduzir o que ele vê na tela do computador.

Em resposta ao progresso tecnológico diário, as agências governamentais vêm analisando e observando as necessidades dos alunos e professores surdos e fornecendo métodos de ensino e assistência técnica para promover a aprendizagem dentro e fora das escolas. Consistente com isso, os recursos de tecnologia assistiva (TA) destacam-se como um bom aliado e promovem práticas inclusivas, aumentando assim a probabilidade de melhoria no processo de ensino e aprendizagem (Souza et al., 2021).

Há vários softwares que os alunos com deficiência auditiva podem usar, tais como o VLibras e o Hand Talk, ambos gratuitos, que permitem a tradução de textos para a LIBRAS. O VLibras foi desenvolvido pela Universidade Federal da Paraíba, em cooperação com o Ministério do Planejamento, com o objetivo de traduzir textos, áudio e vídeo para a língua de sinais (BRASIL, 2021). Possui versões para desktop, smartphone e tablet e pode ser executado nos sistemas operacionais Windows, Android e Linux. O governo federal disponibiliza gratuitamente o VLibras para cidadãos, empresas e suas instituições e instituições no ambiente digital por meio do portal vlibras.gov.br e softwarepublico.gov.br/social/suite.

Também possui uma versão que permite a tradução de vídeos para LIBRAS, VLibras Vídeo. Sua grande vantagem é que pode ser adicionado um dicionário, sendo uma ferramenta colaborativa com um banco de dados público. Também permite que os usuários da Internet instalem plugins diretamente em seus navegadores para traduzir as páginas visitadas. O sistema enfrenta desafios porque tem um logotipo diferente no Brasil, que pode ser interpretado de uma forma em um estado, mas não corresponde à mesma expressão em outro estado. O projeto visa atender as necessidades dos deficientes auditivos de uma forma ampla, permitindo sua integração em qualquer ambiente (Da Mata Caetano et al., 2017).

O VLibras possibilita a tradução de conteúdo digital de qualquer página da web contendo texto, áudio ou vídeo. Pode ser baixado e instalado em computadores, tablets e smartphones. Há também o VLibras Vídeo, em que os usuários podem contribuir enviando frases na língua de sinais. 


\subsection{Software de Edição}

Para Vargas (2007), embora normalmente associada ao lazer e ao entretenimento, a produção de vídeo digital ainda pode ser utilizada como uma atividade de ensino e aprendizagem com grande potencial pedagógico, que ainda precisa ser explorado. A literatura profissional aponta alguns benefícios educacionais, tais como o desenvolvimento do pensamento crítico, a promoção da expressão e da comunicação, o favorecimento de uma visão interdisciplinar, a integração de diferentes capacidades e inteligências, e a valorização do trabalho em grupo, complementa o citado autor.

O processo de produção de um vídeo é dividido basicamente em três etapas. Na pré-produção são feitas a sinopse ou storyline, o argumento, o roteiro e o Storyboard. Em seguida vem a produção e a pós-produção, que inclui a edição e finalização do vídeo, e é nesta fase que o professor fará a junção do vídeo do VLibras com o vídeo da aula.

Talento e técnicas são essenciais para que o vídeo alcance bons resultados. No entanto, para fazer a montagem certa, usar o programa certo pode ajudar. Existem várias opções para os diferentes perfis de editores, e sua escolha depende das habilidades, necessidades e possibilidades de investimento (Baltarejo, 2017). O Quadro 1 apresenta alguns desses softwares.

Também é possível encontrar outros softwares para edição de vídeos, como por exemplo: Openshot, Window Movie Maker, Avidemux, iMovie, Movavi, Filmora, Openshot, Videopad, HitFilm Express, OBS Studio.

Quadro 1. Software de edição.

\begin{tabular}{|c|c|c|}
\hline Softwares & Prós & Contras \\
\hline $\begin{array}{l}\text { Adobe Premiere } \\
\text { Pro }\end{array}$ & $\begin{array}{l}\text { Popular no mercado de trabalho; Funciona mesmo em } \\
\text { computadores com configurações simples; Faz parte de um } \\
\text { pacote de ferramentas integradas. }\end{array}$ & Pagamento mensal \\
\hline Final Cut Pro X & $\begin{array}{l}\text { Interface e usabilidade amigável; Preço acessível e pago sem } \\
\text { mensalidade. }\end{array}$ & Apenas para MAC \\
\hline DaVinci Resolve & Melhor software para coloristas; Versão gratuita completíssima. & $\begin{array}{l}\text { Necessita de um computador com } \\
\text { boa performance }\end{array}$ \\
\hline $\begin{array}{l}\text { Avid Media } \\
\text { Composer }\end{array}$ & $\begin{array}{l}\text { Software extremamente estável; O software mais usado no } \\
\text { cinema internacional. }\end{array}$ & $\begin{array}{l}\text { Interface arcaica de difícil } \\
\text { aprendizagem; Pagamento mensal }\end{array}$ \\
\hline Sony Vegas Pro & $\begin{array}{l}\text { Fácil aprendizagem; Funciona mesmo em computadores com } \\
\text { configurações simples. }\end{array}$ & $\begin{array}{l}\text { Faltam algumas ferramentas de pós } \\
\text { produção }\end{array}$ \\
\hline Edius & Sistema multi câmera e broadcast & $\begin{array}{l}\text { Burocracia de compra; pouco usado } \\
\text { no mercado nacional }\end{array}$ \\
\hline
\end{tabular}

Fonte: Autores (2021).

\section{Percurso Metodológico}

Esta seção apresenta o percurso metodológico adotado para gerar o vídeo com o VLibras. A metodologia descreve as atividades realizadas, os objetos gerados em cada uma das atividades e, também, provê detalhe de como foram realizadas. A seguir, cada uma das atividades é detalhada.

Esta pesquisa pode ser caracterizada como aplicada, qualitativa e de estudo de caso. Aplicada por que "visa resolver problemas concretos" (Laville \& Dionne, 1999, p. 25), qualitativa por que se relaciona “a uma visão de mundo em que há um 
desejo de compreensão subjetiva do homem e seu universo, sendo que os dados utilizados descrevem aspectos relacionados ao objeto de pesquisa de forma enriquecedora" (DIAS, 2010, p. 108). Por fim, um estudo de caso por que investigou os resultados de uma tecnologia assistiva (VLibras) por especialistas em Libras. Estudo de caso como estratégia metodológica porque é vivenciado num contexto real e atual, na busca do como e do porquê de um fenômeno ter ocorrido ou estar ocorrendo (YIN, 2010). Devido à pandemia de Coronavírus (COVID-19), a situação de distanciamento social de nossas vidas ao redor do mundo não pode ser conduzida em pesquisas in loco porque a recomendação da autoridade do órgão de saúde é dar prioridade ao distanciamento social em todas as partes do país. Portanto, para conduzir a presente investigação, foi utilizado além da criação de um vídeo foi utilizado um questionário online com especialistas em Libras.

\subsection{Instalação do Software}

Em termos de implementação, para instalação do VLibras configuramos a função de som do computador para que o Google Document capturasse o som do vídeo ou áudio e gerasse um documento que transcrevesse o áudio em texto. Utilizamos o aplicativo VLibras por meio de smartphones e tablets para ler o texto capturado e fazer imagens em língua de sinais. Ele será inserido no vídeo final como uma tradução em LIBRAS para o vídeo-curso do educador.

Para transcrever áudio em texto para arquivos no Google Docs, mesmo vídeos do YouTube, geralmente se coloca um microfone próximo ao alto-falante, mas isso pode degradar a qualidade do som. No entanto, existe um plugin para o dispositivo de áudio virtual do sistema operacional Windows chamado VB-CABLE. É gratuito e é um cabo virtual, o que reduz a mão de obra e melhora a qualidade. A seguir são apresentados os passos para a realização da configuração do som no computador, captura e transcrição do texto, tradução pelo VLibras e edição final do vídeo.

\section{A) Configuração do som no computador}

1. Entre no site do plugin, https://vb-audio.com/Cable/index.htm. Baixe e descompacte o arquivo;

2. Para a instalação do software, clique em VBCABLE_setup_x64.exe (Figura 1) com o botão direito do mouse, selecione Executar como administrador (1) e siga as instruções de instalação;

Figura 1. Instalação do software.

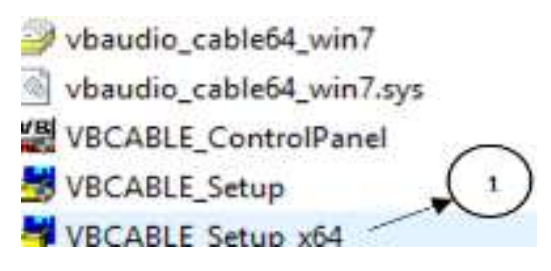

Fonte: Autores (2021).

3. Após a instalação, como mostra a Figura 2, clique no ícone do alto-falante na Barra de Ferramentas Figura 2 (1) com o botão direito do mouse e selecione Abrir Som Figura 2 (2); 
Figura 2. Localização painel do som.

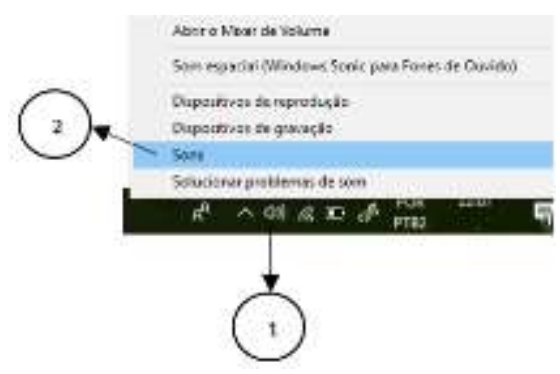

Fonte: Autores (2021).

4. Para a configuração da reprodução do som, a Figura 3, siga os seguintes passos: selecione a Aba Reprodução (1), clique com o lado direito do mouse em CABLE Input (2), e em seguida com lado esquerdo do mouse clique em cima de definir como Dispositivo Padrão (3).

Figura 3. Configurando a reprodução do som.

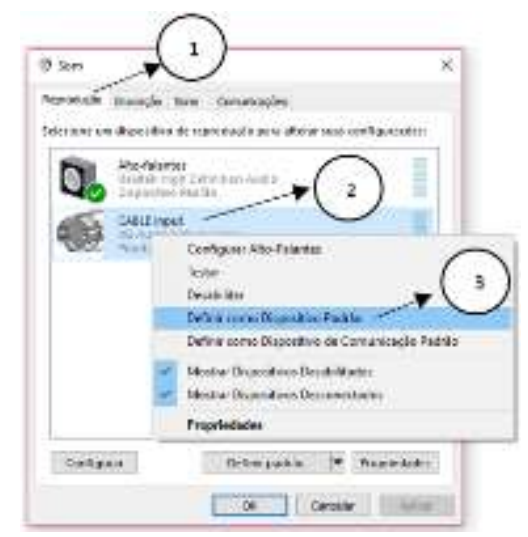

Fonte: Autores (2021)

5. Nesta etapa foi configurado a gravação do som, Figura 4, sendo realizado os seguintes passos: selecione a Aba Gravação Figura 4 (1), clique com o lado direito do mouse em cima CABLE Output Figura 4 (2); em seguida com lado esquerdo do mouse clique em cima de definir como Dispositivo Padrão Figura 4 (3). 
Figura 4. Configurando a gravação do som.

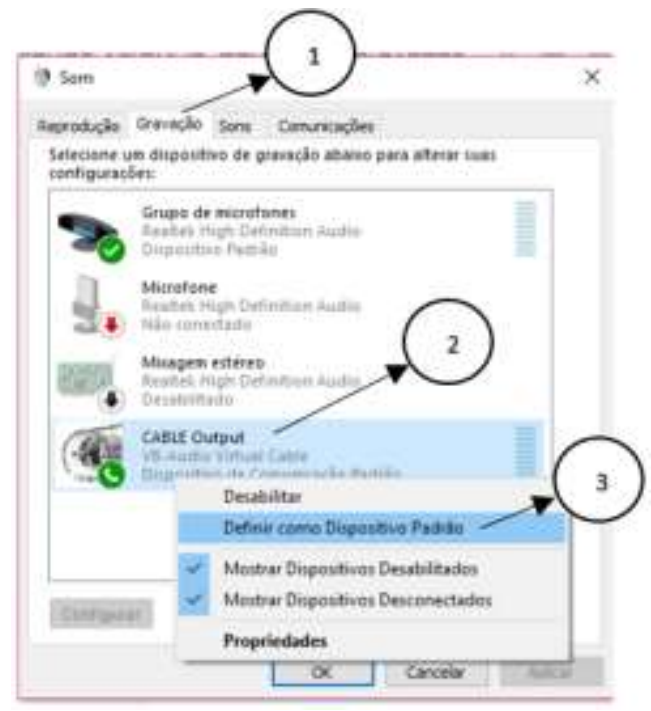

Fonte: Autores (2021).

\section{B) Captura e transcrição do texto}

1. Após as operações serem concluídas, o computador poderá ficar silencioso (sem som), por isso, retorne ao modo selecionado anteriormente e o som voltará a funcionar novamente, porém somente após a captura do som.

2. Abra um novo arquivo no Documentos Google, clique na aba Ferramentas (1), e selecione Digitação por Voz (2), veja a Figura 5 .

Figura 5. Documentos Google Selecionando o microfone.

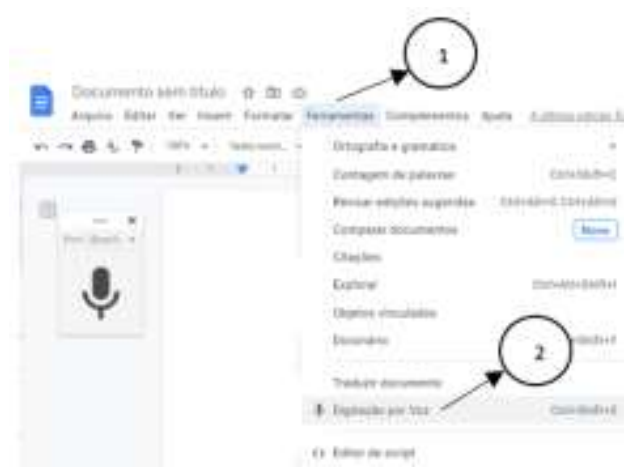

Fonte: Autores (2021).

3. Para execução do arquivo clique em: Figura 6 (1) o arquivo (vídeo ou áudio) que deseja transcrever, que pode estar armazenado localmente ou na Web; veja Figura 6. 
Figura 6. Vídeo que deseja transcrever para texto.

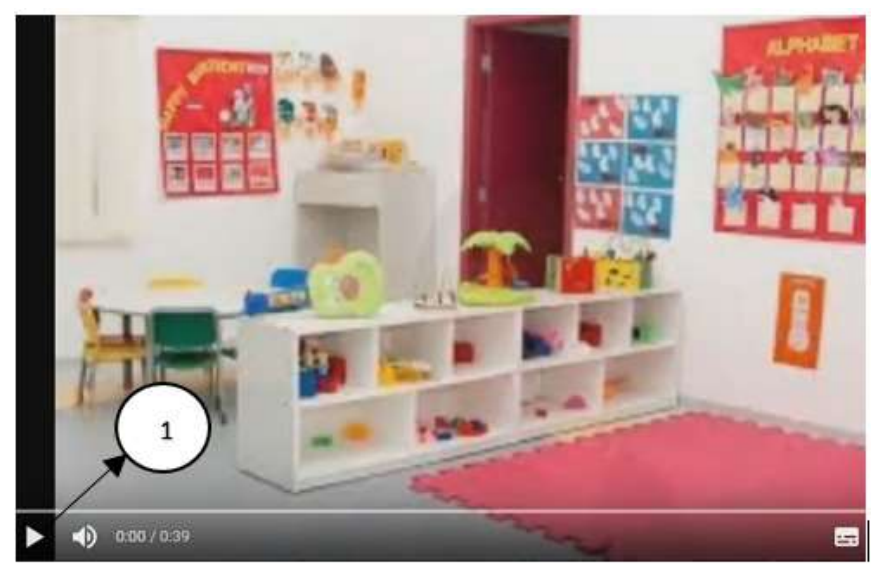

Fonte: Autores (2021).

4. Acesse o Google Docs e clique no ícone do microfone Figura 7 (1) e a transcrição do texto será iniciada Figura 7 (2), conforme mostrado na Figura 7. Finalmente para parar a gravação, clique novamente no microfone Figura 7 (1).

Figura 7. Transcrição do texto no Documentos Google.

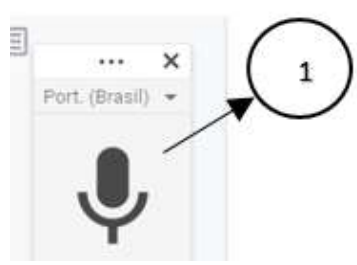

Olá crianças vamos para mais uma aulinha online e vamos ver qual é a novidade de hoje. Ola crianças quero ver quem vai escrever palavra com $B$ bolo boneca banana boi bode bigode e ai aguardo você enviar os parabéns no grupo do WhatsApp hein tchau

Fonte: Autores (2021).

\section{C) Tradução pelo VLibras}

1. Baixe o Aplicativo VLibras (VLIBRAS, 2021) no smartphone e faça a instalação. Selecione e copie o texto do Documentos Google que foi alvo da transcrição. Abra o VLibras e, na tela inicial, clique na opção traduzir texto como visto na Figura 8 (a) (1). Neste momento será exibida a tela de traduzir texto Figura 8 (b), na caixa de texto Figura 8 (b) (2) clique dentro e cole, em seguida clique no botão OK Figura 8 (b) (3). O aplicativo vai exibir a tela Figura 8 (b), clique no botão Traduzir Figura 8 (c) (4). 
Figura 8. Tela inicial do Aplicativo VLibras.
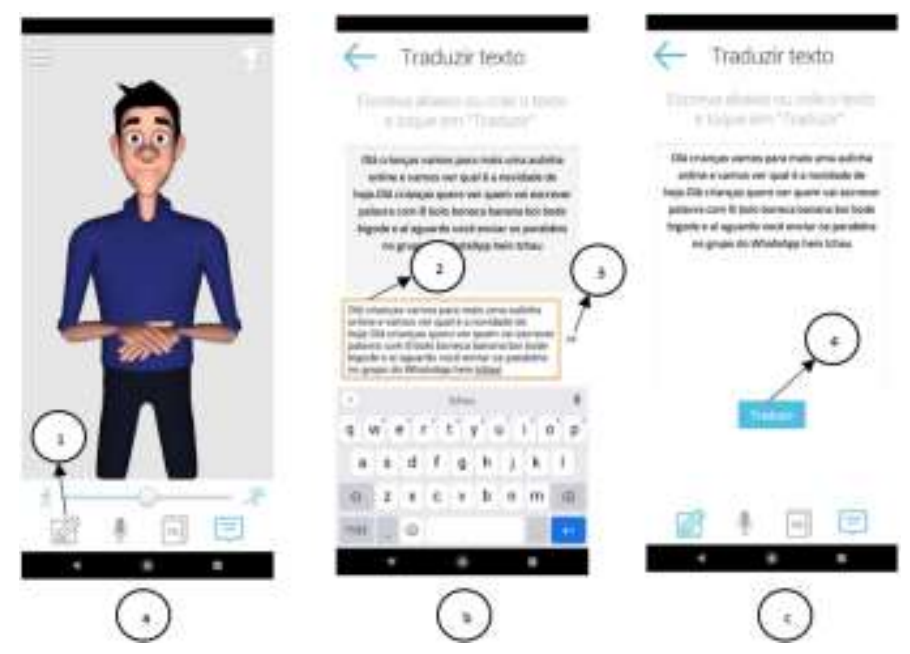

Fonte: Autores (2021).

2. Logo iniciará a tradução, conforme Figura 9 (a) (1); em Figura 9 (b) desta mesma Figura o aplicativo iniciou a tradução, usando símbolos e letras Figura 9 (b) (2). Ao terminar a tradução do texto que foi inserido, o personagem apresenta as mãos conforme Figura 9 (c) (3).

Figura 9. Tela do Aplicativo VLibras traduzindo o texto.
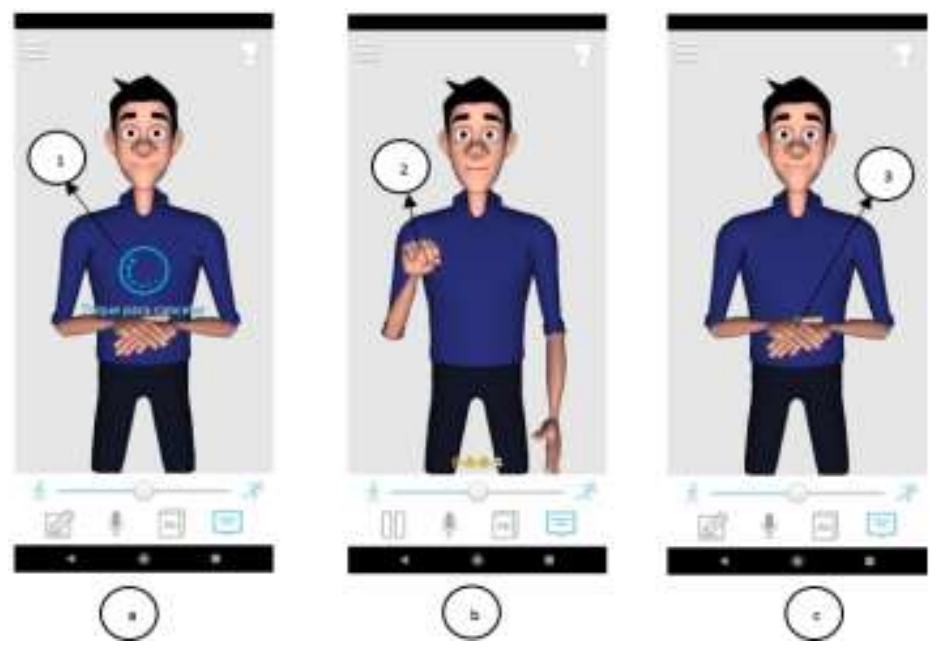

Fonte: Autores (2021).

\section{D) Edição final do vídeo Inserindo o VLibras}

1. Software de gravação e tela do VLibras

É necessário fazer o espelhamento do celular no computador, e para isso, utilizamos o Letsview, que é um software gratuito com funções que permitem espelhar sem fio a tela do seu celular para a TV e o computador. Suporta espelhamento de tela entre vários dispositivos com diferentes sistemas operacionais. Porém é possível utilizar qualquer outro software de espelhamento. Após realizar o espelhamento selecione no celular aplicativo VLibras e execute os passos em ((C) Tradução pelo VLibras). Com isso o aplicativo VLibras estará pronto para iniciar a tradução. Mas antes de iniciar a gravação é preciso criar uma tela que fará a simulação do Chroma Key, que é um recurso de efeito visual que envolve a colocação de uma 
imagem sobre outra, cancelando as cores padrão (como verde ou azul). Utilizamos um aplicativo de apresentação com PowerPoint ou Impress, deixando a tela do aplicativo de apresentação ampliada. Por fim, coloque o VLibras na frente, como visto na Figura 10.

Figura 10. Tela para utilizar o Chroma Key na edição.

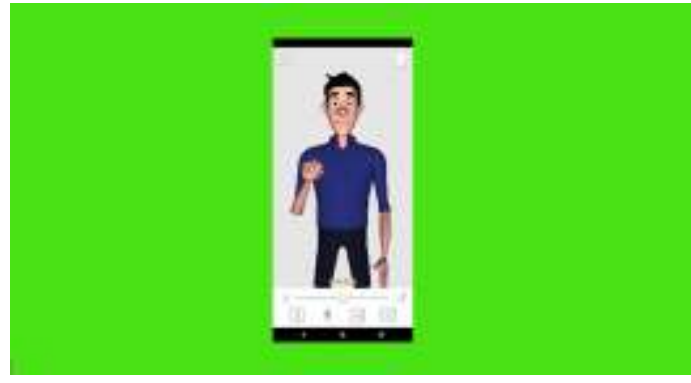

Fonte: Autores (2021).

Para capturar a tela do computador, que estará pronta para iniciar o software VLibras, utilize um software de captura de tela. Dentre os vários existentes, escolhemos o Action!, que é uma ferramenta de gravação de vídeo que permite capturar imagens dinâmicas na área de trabalho do computador com alta definição e som.

Ao término da tradução do VLibras paramos a captura da tela pelo software Action! e foi gerado um arquivo. Para finalizar o vídeo utilizamos o software de edição Adobe Premiere Pro, com listado no Quadro 1 que trata dos softwares de edição para vídeo. Neste momento foram importados os dois vídeos, o da vídeo-aula e o do VLibras com Chroma Key. No vídeo VLibras é aplicado o efeito Chroma Key na ferramenta de edição. Ao final, ainda é preciso sincronizar o tempo de início da fala do professor na vídeo-aula com o início da tradução do vídeo do VLibras. O resultado final é visto na Figura 11.

Figura 11. Vídeo finalizado.

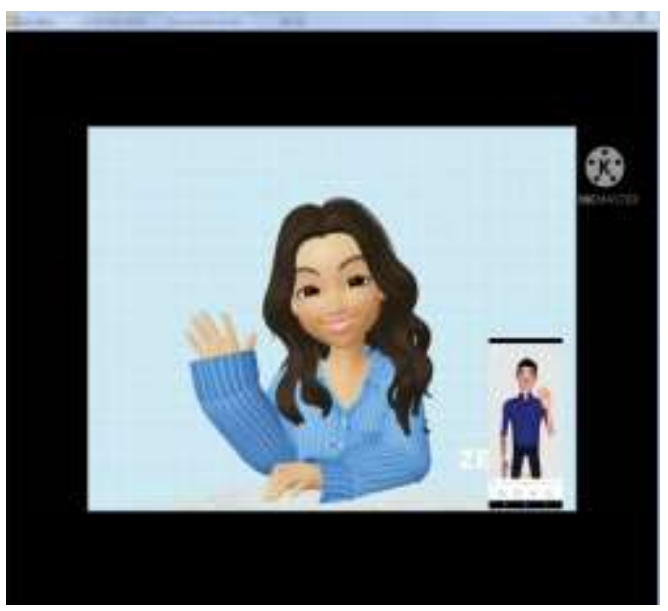

Fonte: Autores (2021).

\section{Resultados e Discussão}

Todos os estudos mencionados nos trabalhos relacionados evidenciam que os recursos da tecnologia assistiva vem a colaborar com o ensino aprendizagem dos alunos com surdez. Castro (2019) utilizou o VLibras e demonstrou que esse recurso 
educacional contribui positivamente para melhorar a comunicação de alunos surdos-mudos. Os resultados obtidos durante este estudo apontam para a necessidade de inclusão, que é um direito dos alunos com necessidades especiais. E com o auxílio de tecnologias assistivas como o VLibras é possível preparar vídeo-aulas. Também foi observado por Souza et al. (2021) A julgar pela conclusão da pesquisa, conclui-se que o VLibras e WIKILIBRAS, promovem práticas socioeducativas inclusivas e essas duas ferramentas digitais são úteis para os professores usarem com alunos surdos em sala de aula, aumentando suas chances de integração.

Em resposta ao questionário online submetido ao grupo de especialistas (um técnico e três tradutores/intérpretes) obtivemos algumas respostas sobre o aplicativo VLibras para tradução do vídeo como a Figura 11. Com relação à transmissão das informações pretendidas, houve um consenso de que isso foi possível, mas como o respondente 2 comentou, "a professora só fala palavras sem contextos", ou seja, talvez em contextos conversacionais mais complexos a ferramenta poderia ser menos eficiente.

Como principais problemas ou dificuldades na tradução de um vídeo para alunos surdos utilizando o VLibras, vale salientar a fala do tradutor 02, que afirmava que o VLibras "Não compreendem a mensagem, só palavras soltas. Muita datilologia. A Libras tem como parâmetro a expressão facial que dá intensidade ao sinal, mas o aplicativo não apresenta as expressões faciais e corporais." Além disso, como aponta o tradutor 01, o VLibras "Não realiza tradução, tornando incompreensível o contexto." Outro ponto negativo identificado por todos os especialistas foi o fato de que, durante a edição do vídeo, o avatar não pode seguir as legendas do vídeo ao mesmo tempo. O aplicativo continuava a traduzir mesmo depois que o vídeo havia terminado, sendo necessário uma intervenção da timeline para estes ajustes de sincronia, de forma manual.

Há evidências de que ao se utilizar o software VLibras para traduzir texto em símbolos, o software não consegue reconhecer algumas palavras e, por isso, ele precisa soletrar, tornando o vídeo mais longo. Ao se ajustar o tempo, pode ocorrer dele ficar mais rápido, podendo tornar a leitura e interpretação mais difícil. Portanto, recomenda-se que, no texto que foi transcrito no Google Documentos, o professor faça a substituição de palavras no diminutivo ou no aumentativo por outra palavra com o mesmo significado. Como por exemplo: para a palavra casinha, faça a substituição por casa pequena. Neste mesmo sentido, houve a necessidade de ajustar o tempo de execução da timeline do programa editor de vídeo. Devido ao fato de o software VLibras não acompanhar a fala do professor, sendo mais rápido ou lento, fizemos cortes em pontos de pausa da voz, para realizar esta sincronização.

No entanto, ainda há muito trabalho a ser feito nesse processo de inclusão. Para os professores, foi evidenciado que é possível incluir em seus vídeos recursos da tecnologia assistiva, e contribuir para a promoção do ensino aprendizagem. Mas ao perceberem falhas na tradução, podem avaliar se as contrapartes linguísticas estão realmente prejudicadas. Com isso os aspectos que auxiliam a comunicação entre o surdo e o ouvinte foram completados e, mesmo que haja dificuldades. Sendo utilizado apenas um software de TA na preparação do vídeo nesta pesquisa e avaliação por um técnico em LIBRAS para análise da efetividade na transmissão da informação. Como trabalho futuro é fazer vídeos mais longos, e com outras ferramentas de TA para uma análise com alunos e técnicos em LIBRAS.

No caso desta investigação, houve utilização dos recursos tecnológicos digitais como: (software VLibras, captura de áudio e gravação de tela) vindo a contribuir para o processo de ensino aprendizagem na construção de vídeo com aula inclusiva. No entanto, não devemos esquecer da importância do tradutor e intérprete de LIBRAS no processo de ensino aprendizagem dos alunos que necessitem deste apoio. Os recursos e ferramentas técnicas disponíveis para enriquecer as videoaulas disponibilizadas neste momento da pandemia do Covid-19. 


\section{Considerações Finais}

A tecnologia assistiva é um campo do conhecimento com características interdisciplinares, incluindo produtos, recursos, metodologias, estratégias, práticas e serviços que visam promover a atividade e a participação de pessoas com deficiência ou pessoas com mobilidade reduzida, visando a sua autonomia e independência. A julgar por essa característica a TA apresenta-se como mais um recurso para utilização da LIBRAS, fazendo com que ela se torne mais uma aliada no processo geral de ensino-aprendizagem.

A pesquisa buscou investigar o uso da tecnologia assistiva VLibras, que envolve um conjunto de vídeos em Língua Brasileira de Sinais (LIBRAS). Com uso do VLibras e de um software de edição de vídeo foi realizada a edição e, como resultado, na aula da professora foi inserido um recurso para alunos que necessitarem da LIBRAS. É sugerido que em pesquisas futuras outras ferramentas de tecnologia assistiva sejam analisadas e ainda seja apresentado o vídeo final para alunos surdos realizarem suas observações.

\section{Agradecimentos}

Os autores agradecem ao CNPq, UFRPE e à FACEPE pelo apoio na realização desta pesquisa.

\section{Referências}

Araújo, E. M., Teves, R., Machado, R., \& Rofino, R. (2021). Impacto da Tecnologia Assistiva em indivíduos com Dislexia: uma Revisão Sistemática. https://psyarxiv.com/hk9y6/. https://doi.org/10.31234/osf.io/hk9y6

Baltarejo, B. (2021). Os 7 melhores softwares de edição de vídeos, 2017. https://www.avmakers.com.br/blog/os-7-melhores-softwares-de-edicao-de-videos

BRASIL. Constituição (2001), Capítulo III - Da educação, da Cultura e do Desporto. Seção I da Educação, art. 205. http://portal.mec.gov.br/setec/arquivos/pdf/LegisBasica.pdf

Brasil. Lei no 10.436, de 24 de abril de 2002. Dispõe sobre a Língua Brasileira de Sinais-Libras e dá outras providências. Diário Oficial da União, 2002.

Brasil. Governo Digital. VLibras. Acesso em: 13 jul. 2021. https://www.gov.br/governodigital/pt-br/vlibras/

Braga, M. D. P. (2020). Tradução Automática para Língua Brasileira de Sinais: um estudo sobre a percepção de qualidade e uso do serviço. Disponível em: <https://repositorio.ufsc.br/handle/123456789/219816> Acesso em: 17 jul. 2021.

Bersch, R., \& Tonolli, J. C. (2006). Introdução ao conceito de Tecnologia Assistiva e modelos de abordagem da deficiência. Bengala Legal, 25.

Bersch, R. Introdução à tecnologia assistiva. (2021) https://www.assistiva.com.br/Introducao_Tecnologia_Assistiva.pdf

Bersch, R. (2008). Introdução à tecnologia assistiva. Porto Alegre: CEDI, 21.

Bogas, J. V. (2014). A história da Libras, a Língua Brasileira de Sinais. Hand Talk, http://blog.handtalk.me/historia-lingua-de-sinais/

Castro, T. M. L. (2019). Ensinando as quatro operações básicas da matemática no ensino médio, usando o aplicativo VLibras. TCC. Graduação em Licenciatura em Computação - Licenciatura - Universidade do Estado do Amazonas, Manaus, 2019. https://bit.ly/3wKFAi0

CAT - Comitê de Ajudas Técnicas. (2006). Portaria nº 142, de 16 de novembro de 2006. https://www.assistiva.com.br/tassistiva.html

Da Mata Caetano, M. S.; Passos, M. L. S. (2017). A Utilização Dos Softwares Vlibras E Hand Talk No Processo De Inclusão De Alunos Com Deficiência Auditiva Em Uma Escola Regular. Anais... Anais do IV Congresso Regional de Formação e EAD. Vitória-ES. 2017. https://www.researchgate.net/profile/marize-passos/publication/322924146_a_utilizacao_dos_softwares_vlibras_e_hand_talk_no_processo_de_inclusao_

de_alunos_com_deficiencia_auditiva_em_uma_escola_regular/links/5a76816f45851541ce588e93/a-utilizacao-dos-softwares-vlibras-e-hand-talk-no-processo -de-inclusao-de-alunos-com-deficiencia-auditiva-em-uma-escola-regular.pdf

Da Silva, E. G. N., \& Cardoso, C. D. N. A. (2021). A importância do uso da tecnologia assistiva na educação de surdos. Research, Society and Development, 10(3), e28410313153-e28410313153. https://doi.org/10.33448/rsd-v10i3.13153

De Lima, R. L. Q., Galasso, B. J. B., \& Thompson, C. E. M. (2021). As contribuições das tecnologias assistivas na Educação Profissional e Tecnológica na modalidade EAD. Brazilian Journal of Development, 7(1), 1692-1704. https://doi.org/10.34117/bjdv7n1-116

Dias, M. T. (2010) Construção do Conhecimento e Metodologia da Pesquisa. Natal: [s.n.], 2010. 256p.

Edyburn, D. L. (2015). Expanding the use of assistive technology while mindful of the need to understand efficacy. In: D. Edyburn (Ed.). Efficacy of assistive technology interventions (pp. 1-12). Emerald Group Publishing Limited. Expanding the Use of Assistive Technology While Mindful of the Need to Understand Efficacy 
Research, Society and Development, v. 10, n. 12, e385101220720, 2021

(CC BY 4.0) | ISSN 2525-3409 | DOI: http://dx.doi.org/10.33448/rsd-v10i12.20720

Federici, S. \& Scherer, M. J. (2018). The Assistive Technology Assessment Process Model and Basic Definitions. In S. Federici; M. J. Scherer (Eds.). Assistive Technology Assessment Handbook (2nd ed., pp. 1-9). Taylor \& Francis Group.

Laville, C.; Dionne, J. (1999). A construção do saber - manual de metodologia da pesquisa em ciências humanas. Porto Alegre-RS: Artmed. 1999. https://elizabethruano.com/wp-content/uploads/2016/08/laville-dionne-2008-cic3aancias-humanas-e-sociedade.pdf

Ramos, T. S. (2021). Metodologias de ensino da língua brasileira de sinais-LIBRAS: proposta de ensino para ouvintes por meio de produções literárias surdas. http://hdl.handle.net/11612/2768

Sartoretto, M. L. \& Bersch, R. (2017) Assistiva: tecnologia e educação. https://www.assistiva.com.br/tassistiva.html

Souza, A. M., \& Rech, T. L. (2021). A utilização de recursos de tecnologia assistiva para crianças surdas. Seminário Nacional de Educação Inclusiva, 1(1). https://portaleventos.uffs.edu.br/index.php/SENEI/article/view/15351/10016

Vargas, A.; Da Rocha, H. V.; Freire, F. M. P. (2007). Promídia: produção de vídeos digitais no contexto educacional. RENOTE-Revista Novas Tecnologias na Educação, v. 5, n. 2, 2007. https://doi.org/10.22456/1679-1916.14199

Vlibras. (2010) Governo Digital. VLibras. https://www.gov.br/governodigital/pt-br/vlibras

Yin, R. K. (2010) Estudo de caso: planejamento e métodos. 4 ed. Porto Alegre: Bookman editora. 